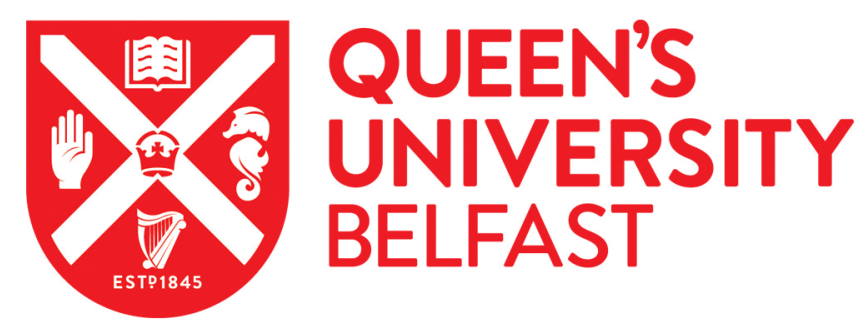

\title{
Ectopic bone formation in rapidly fabricated acellular injectable dense collagen-Bioglass hybrid scaffolds via gel aspiration-ejection
}

Miri, A. K., Muja, N., Kamranpour, N. O., Lepry, W. C., Boccaccini, A. R., Clarke, S. A., \& Nazhat, S. N. (2016). Ectopic bone formation in rapidly fabricated acellular injectable dense collagen-Bioglass hybrid scaffolds via gel aspiration-ejection. Biomaterials, 85, 128-141. https://doi.org/10.1016/j.biomaterials.2016.01.047

\section{Published in:}

Biomaterials

\section{Document Version:}

Peer reviewed version

Queen's University Belfast - Research Portal:

Link to publication record in Queen's University Belfast Research Portal

Publisher rights

( 2016 Elsevier. This manuscript version is made available under the CC-BY-NC-ND 4.0 license http://creativecommons.org/licenses/by-ncnd/4.0/ which permits distribution and reproduction for non-commercial purposes, provided the author and source are cited.

\section{General rights}

Copyright for the publications made accessible via the Queen's University Belfast Research Portal is retained by the author(s) and / or other copyright owners and it is a condition of accessing these publications that users recognise and abide by the legal requirements associated with these rights.

Take down policy

The Research Portal is Queen's institutional repository that provides access to Queen's research output. Every effort has been made to ensure that content in the Research Portal does not infringe any person's rights, or applicable UK laws. If you discover content in the Research Portal that you believe breaches copyright or violates any law, please contact openaccess@qub.ac.uk. 
Ectopic bone formation in rapidly fabricated acellular injectable dense collagenBioglass hybrid scaffolds via gel aspiration-ejection

Amir K. Miri ${ }^{1}$, Naser Muja ${ }^{1}$, Neysan O. Kamranpour ${ }^{1}$, William C. Lepry ${ }^{1}$, Aldo R. Boccaccini $^{2}$, Susan A. Clarke ${ }^{3}$, Showan N. Nazhat ${ }^{1 *}$

${ }^{1}$ Department of Materials and Mining Engineering, McGill University, Montreal, QC, H3A 0C5, Canada

${ }^{2}$ Institute of Biomaterials, University of Erlangen-Nuremberg, Cauerstr. 6, D-91058 Erlangen, Germany

${ }^{3}$ School of Nursing and Midwifery, Queen’s University Belfast, Belfast, BT9 7BL, UK

* Corresponding Author:

Showan N. Nazhat

Department of Mining and Materials Engineering

McGill University

3610 University Street

Montreal, QC H3A 0C5

Email: showan.nazhat@mcgill.ca 


\section{ABSTRACT}

Gel aspiration-ejection (GAE) has recently been introduced as an effective technique for the rapid production of injectable dense collagen (IDC) gel scaffolds with tunable collagen fibrillar densities (CFDs) and microstructures. Herein, a GAE system was applied for the advanced production and delivery of IDC and IDC-Bioglass ${ }^{\circledR}$ (IDC-BG) hybrid gel scaffolds for potential bone tissue engineering applications. The efficacy of GAE in generating mineralizable IDC-BG gels (from an initial 75-25 collagen-BG ratio) produced through needle gauge numbers 8G (3.4 mm diameter and $6 \mathrm{wt} \%$ CFD) and 14G (1.6 mm diameter and $14 \mathrm{wt} \%$ CFD) was investigated. Second harmonic generation (SHG) imaging of as-made gels revealed an increase in collagen fibril alignment with needle gauge number. In vitro mineralization of IDC-BG gels was confirmed where carbonated hydroxyapatite was detected as early as day 1 in simulated body fluid, which progressively increased up to day 14 . In vivo mineralization of, and host response to, acellular IDC and IDC-BG gel scaffolds were further investigated following subcutaneous injection in adult rats. Mineralization, neovascularization and cell infiltration into the scaffolds was enhanced by the addition of BG and at day 21 post injection, there was evidence of remodelling of granulation tissue into woven bone-like tissue in IDC-BG. SHG imaging of explanted scaffolds indicated collagen fibril remodelling through cell infiltration and mineralization over time. In sum, the results suggest that IDC-BG hybrid gels have osteoinductive properties and potentially offer a novel therapeutic approach for procedures requiring the injectable delivery of a malleable and dynamic bone graft that mineralizes under physiological conditions.

Keywords: dense collagen; hydrogel; hydroxyapatite; osteoinduction, subcutaneous injection; ectopic bone formation 


\section{Introduction}

Current bone repair techniques include the implantation of autologous, allogeneic, or prosthetic materials [1]. Although these approaches achieve a degree of functional restoration, they possess inherent limitations, such as donor-site morbidity and unpredictable graft resorption $[1,2]$. Therefore, there is an ever-increasing demand for new biomaterials for the replacement, construction or augmentation of bone. In particular, there is a need for injectable, and bioactive materials for bone regeneration $[1,3,4]$.

Hydrogels of type I collagen, the most abundant protein in the body and the main organic component of bone, have excellent biocompatibility, can form physiologically relevant scaffolds and can be injectable. Collagen is widely applied for the engineering of scaffolds for potential clinical applications in regenerative medicine including bone replacement and void filling [1, 5]. However, highly-hydrated collagen (HHC) hydrogels are rapidly absorbed in vivo, suffer from weak mechanical properties, and more importantly are difficult to mineralize under physiological conditions [6], thus limiting their usage for bone tissue engineering. On the other hand, collagen gel hybridization with organic factors that favour osteoid formation, such as recombinant bone morphogenetic proteins have shown mixed results due to suboptimal local growth factor concentrations as well as non-specific cellular activity [7]. In contrast, the incorporation of inorganic bioactive glasses has been shown to increase bioactivity and mineralization, control scaffold degradation rate, and improve the structural integrity of collagen scaffolds [8, 9]. The commercially available bioactive glass $45 \mathrm{~S} 5$ Bioglass ${ }^{\circledR}$ (BG) of the composition (45) $\mathrm{SiO}_{2}-$ (24.5) $\mathrm{Na}_{2} \mathrm{O}-(24.5) \mathrm{CaO}-(6) \mathrm{P}_{2} \mathrm{O}_{5}$ (wt\%), has been proven to favour local deposition of carbonated hydroxyapatite (CHA) in physiological environments [10]. In a recent review, Hench and 
Greenspan [10] described the mechanism for the collagen-BG interface created by the CHA crystals which increases the fracture toughness of the BG-bone interface.

Various techniques have been used to fabricate collagen-BG composite scaffolds [11], such as freeze-drying [12], surface coating through immersion in a suspension of BG $[8,13]$, and the pipetting of collagen-BG solutions onto collagen sponges [14]. While highly porous threedimensional scaffolds are produced through these approaches, the necessities of extended processing steps limit their ease of delivery. It has also been shown that native osteoid mimicking scaffolds can be rapidly generated by plastically compressing prefabricated HHC-BG hybrid gels [9, 15, 16]. Through a brief application of a unidirectional compressive stress [17], the collagen fibrillar density (CFD, i.e., dry to wet weight ratio) of HHC-BG hybrid gels is significantly increased for improved mechanical, biological and microstructural properties [9, 15]. However, the resultant scaffolds are planar-shaped and require rolling and material trimming to yield implantable, cylindrical-shaped constructs that match the dimensions of osseous defects which are often irregular in volume. Furthermore, there is still a need for introducing a higher degree of control on the microstructure and more feasibility for clinical injections of scaffolds [11].

Recently, a gel aspiration-ejection (GAE) technique was developed for the production of injectable dense collagen (IDC) gel scaffolds with tunable CFDs and microstructures [18]. Through the application of pressure differentials, GAE initially draws prefabricated HHC hydrogels into a blunt needle, simultaneously imparting compaction and anisotropy on the gels, which are then ejected to generate IDC gels. These rapid and tunable microstructural changes of more stable collagen hydrogels may enable broad clinical applications. To this end, the efficacy of the GAE technique, which allows for the hybridization of collagen gels for increased 
functionalization [18], was investigated in generating IDC gel scaffolds incorporated with BG microparticles (IDC-BG) for controllable mineralization and potential applications in bone tissue engineering. Furthermore, in order to ultimately ease IDC-BG translation towards clinical applications, a GAE system was developed for the advanced production and delivery of IDC gels. Therefore, the aim of this study was to assess the potential clinical feasibility of the GAE system in generating IDC-BG hybrid gel scaffolds of distinct microstructural properties (i.e., collagen fibrillar density and extent of alignment) imparted by two different needle gauge sizes. The potential clinical outlook for in situ bone defect injections was determined by analyzing the physiological mineralization of the gel scaffolds both in vitro and in vivo by immersion in simulated body fluid (SBF) and subcutaneous injection in adult rats, respectively. Along with assessing host responses, the latter model was chosen to investigate any possible osteoinductive properties of IDC-BG hybrids as a prelude to their implantation in a bone defect model.

\section{Materials and Methods}

\section{Fabrication of IDC-BG gel scaffolds}

Bioactive glass particles (of a similar formulation to Bioglass ${ }^{\circledR}$ 45S5) with an average diameter of 7.0 $\pm 4.1 \mu \mathrm{m}$ were dispersed in 10x-concentrated Dulbecco’s Modified Eagle Medium (10x DMEM; Sigma Aldrich, Canada) by sonication ( 5 min) to produce a BG-DMEM solution with pre-defined mass ratios, as schematically depicted in Fig. 1A. Bovine dermis derived type-I collagen (6 $\mathrm{mg} / \mathrm{mL}$, in acidic solution, Collagen Solutions Ltd, UK) was added to the BGDMEM solution at a 4:1 ratio to yield a final collagen concentration of $4.8 \mathrm{mg} / \mathrm{mL}$. CollagenDMEM solution served as the control solution (i.e., 0\% BG). Following neutralization using $\mathrm{NaOH}$, a standardized IDC processing method was established using the 48-well culture plate (Costar Corp, USA) to ensure the reproducible production of sterile IDC gels via GAE. Aliquots 
of $1.5 \mathrm{~mL}$ of the solution were initially fibrilized in individual wells by incubating at $37^{\circ} \mathrm{C}$ for 30 min to produce precursor HHC (with and without BG) gels. IDC and IDC-BG gels were produced through the GAE system, which consisted of a pressure delivery device commonly used in angioplasty and kyphoplasty procedures (B. Braun Medical Inc., Germany) to apply pressure differentials; a syringe to introduce an incompressible fluid necessary for the ejection process (Fisher); two Luer lock valves that are attached in series to the Luer lock tip of the pressure delivery device to control flow direction (Fisher); and interchangeable blunt stainlesssteel needles (Hamilton Co., USA) used to dictate aspiration extent (Fig. 1B-C).

To assess the consistency of the fabrication process, IDC-BG gel scaffolds were generated using four standard gauge numbers from 8-14G and two BG concentrations (Table 1; $N=3$ in each group). Gel CFD values were determined by calculating the ratio of dry to wet weight (assuming that no collagen was lost during GAE). Scaffolds were weighed before and after freeze-drying (VirTis Benchtop, USA) at $-105{ }^{\circ} \mathrm{C}$ and 100 mtorr applied for $24 \mathrm{~h}$. Although higher needle gauges (i.e., smaller diameters) yielded higher CFD values, the scaffolds exhibited less retention of BG microparticles. BG particle loss was noteworthy at 50-50 collagen-BG ratio, which may be due to a greater pressure gradient imposed by the narrower needle pathway during the aspiration-ejection process. The collagen and BG densities $\left(1.41\right.$ and $2.73 \mathrm{~g} / \mathrm{cm}^{3}$, respectively) were implemented into the rule of mixtures to calculate the BG volume percent $[19$, 20]. IDC and IDC-BG gels from a 75-25 initial collagen-BG ratio and produced through 8G and $14 \mathrm{G}$ needle gauge numbers, the opposite ends of the current GAE processing spectrum, were selected for further analysis.

\section{In vitro and in vivo mineralization}


Kokubo's protocol for SBF [21] was used to investigate the mineralization potential of IDC and IDC-BG gel scaffolds, in vitro. The scaffolds were conditioned in $50 \mathrm{~mL}$ of SBF (i.e., a ratio of $1: 7 \mathrm{mg} / \mathrm{mL}$ ) at $37^{\circ} \mathrm{C}$ and collected after $6 \mathrm{~h}$, and at days $1,3,7$, and 14 . The samples were washed twice using deionized water immediately prior to chemical and structural characterizations. SBF solution was freshly prepared and exchanged at 48-h intervals. Two repeats were performed for each scaffold type and time point.

In vivo mineralization and bioactivity tests of IDC and IDC-BG gel scaffolds were performed at the animal facility of McGill University. Surgical procedures were performed according to an experimental research protocol (\#2013-7384; approved by the McGill University Animal Care Committee). In turn, eighteen adult male Fischer-344 rats (250-300 g, Harlan) were transferred to an induction chamber and anesthetized using isoflurane inhalation (2-4\% isofluorane gas vaporized in $\mathrm{O}_{2}$ ). Animals were then transferred to a warm heating pad and ophthalmic ointment was applied to each eye to protect the conjunctival membrane in the absence of a blink reflex. Anesthesia was maintained using $0.5-2 \%$ isoflurane delivered through a silicone nose cone and waste gases were scavenged using a coaxial Bain circuit and an activated charcoal canister. Once a surgical depth of anesthesia was confirmed, a $2 \mathrm{~cm}$ wide by $4 \mathrm{~cm}$ long area of fur immediately caudal of each scapula was trimmed using a Wahl Pocket Pro clipper followed by the application of depilatory cream for approximately 1 min to remove residual fur. The skin was aseptically prepared by alternate swabbing of chlorhexidine and 70\% ethanol using saturated surgical gauze and ethanol wipes, respectively. With a disposable \#15 scalpel blade, a small $0.5-1.0 \mathrm{~cm}$ incision was created along each flank. Lidocaine was topically administered along the border of each incision to minimize pain. The fascia between the skin and underlying muscle was then blunt dissected to create a subcutaneous space approximately 2 
$\mathrm{cm}$ rostral and $2 \mathrm{~cm}$ caudal to the incision to accommodate needles used to inject IDC and IDCBG gel scaffolds (Fig. 1D).

Two identical gel scaffolds (8G or $14 \mathrm{G}$ needle gauge numbers) were injected into each flank for a total of four scaffolds per animal (Table 2; number of rats: $N=3$ for IDC and $N=6$ for IDC-BG groups). The scaffolds containing either vehicle (i.e., DMEM) or BG were prepared under sterile conditions by aspiration through the bore of either an 8G or $14 \mathrm{G}$ needle. Skin incisions were immediately closed using 3-0 Ethilon nylon suture, and Carprofen was administered subcutaneously using a weight related dose $(5-10 \mathrm{mg} / \mathrm{kg})$ to control post-operative pain. The animal was revived by oxygen inhalation, returned to its cage and monitored for full ambulatory recovery. At either day 7 or 21 post-surgery, animals were euthanized by asphyxiation using $\mathrm{CO}_{2}$ inhalation followed by pneumothorax. The scaffolds were carefully harvested from the injection site in a necropsy suite for either X-ray micro-tomography followed by histological evaluation (formalin fixation), or chemical analysis (snap frozen in liquid nitrogen).

\section{Morphological characterization}

Nonlinear laser scanning microscopy was performed to investigate the ultra-structure of the IDC and IDC-BG gels. This technique allows in-depth imaging of the gel scaffolds, thus eliminating the artefacts associated with edge effects [22]. The scaffolds were fixed in $4 \%$ formaldehyde solution and embedded in optimal cutting temperature compound (Sakura Finetek, USA) before sectioning with a cryostat microtome into $50 \mu \mathrm{m}$ slices within the bulk of the gel scaffolds and placed between two coverslips. The slides were placed on a Leica multiphoton confocal fluorescence imaging system (SP8; Leica, Germany), equipped with a 63x Leica glycerin-immersion objective. The laser excitation wavelength was set at $830 \mathrm{~nm}$, and the 
scattered signals were collected in the forward direction using one transmitted light photomultiplier tube (low-pass filter; $410 \mathrm{~nm}$ ). The second harmonic generation (SHG) emission of the collagen under excitation was captured.

The Leica system, equipped with synchronized dual-mode imaging of thick samples in both SHG and an infrared scanning gradient contrast modality (Dodt channel) allowed for investigating the different underlying structures in the sectioned IDC-BG gels by phase contrast, and provided an indication of the spatial distribution of the BG particles [23]. A similar concept was also used to track the progress of mineralization and cell infiltration within the IDC and IDC-BG scaffolds.

Scanning electron microscopy (SEM) was used to characterize the progression of IDC-BG scaffolds mineralization as a function of time in SBF. Samples were prepared by fixation in a solution of $4 \%$ formaldehyde at $4{ }^{\circ} \mathrm{C}$. Samples were rinsed three times with distilled water, and a serial dehydration process was carried out by sample immersion in 30,50 , and $70 \%$ ethanol solutions for 10 min periods followed by 80 and 90\% ethanol for 15 min each, and 100\% ethanol for 30 min prior to transfer to hexamethyldisilazane (Sigma Aldrich, Canada) for drying in chemical fume hood. Following gold coating, the samples were observed by SEM (FEI Inspect F50, FEI, USA) at an acceleration voltage of 5-10 kV. For elemental analysis, energy dispersive spectroscopy (EDS) using an attached EDAX and a TEAM EDS Analysis System was performed at $5 \mathrm{kV}$ on 3 unique surfaces areas.

\section{Chemical characterization}

Attenuated total reflectance-Fourier transform infrared (ATR-FTIR) spectroscopy was performed to investigate the structural properties of the scaffolds. The samples were freeze-dried at $-105^{\circ} \mathrm{C}$ and 100 mtorr for $24 \mathrm{~h}$, and then flattened using a pneumatic (10 bar) compressor to 
enable direct contact between the sample and IR prism, and to preserve consistency among different samples with distinct CFDs. ATR-FTIR spectra were collected through a Spectrum 400 (Perkin-Elmer, USA) using a resolution of $2 \mathrm{~cm}^{-1}$, an infrared range of $4000-650 \mathrm{~cm}^{-1}$ and 64 scans. The data were normalized to the absorbance of amide I in collagen (at $1643 \mathrm{~cm}^{-1}$ ).

X-ray diffraction (XRD) patterns of the scaffolds were analyzed using a Bruker D8 Advanced XRD (Bruker AXS Inc., USA) from 3 to $90^{\circ} 2 \theta$ at $40 \mathrm{kV}, 20 \mathrm{~mA}$. Three $30^{\circ}$ - frames were recorded for a 15 min period and then merged using EVA software (Bruker). The phase composition was characterized through a comparison of acquired diffractographs and peaks identified in the International Centre for Diffraction Data (ICDD) database.

\section{Micro-computed tomography}

Micro-computed tomography (micro-CT) analysis was used to investigate the extent of mineralization of IDC and IDC-BG gels at days 7 and 21 post subcutaneous injection. Samples were scanned with a SkyScan 1172 (Aartselaar, Belgium) scanner with tube settings of $50 \mathrm{kV}$ and $200 \mu \mathrm{A}, 5.0 \mu \mathrm{m}$ pixel resolution, four-frame averaging, rotation step of 0.50 degrees, and no filter. The scan times were approximately $1 \mathrm{~h}$ per sample. Following scanning, a transverse reconstruction was performed using a beam hardening correction of $80-100 \%$, a ring artifact correction of 15 and a proper misalignment correction (NRecon software, SkyScan), to generate $2356 \times 2356$ pixel cross-sectional images. As-made IDC and IDC-BG gel scaffolds were scanned to provide a baseline for the beam attenuation (AT) calibration. Two-dimensional analysis (software CTAn, SkyScan) was carried out on a threshold of 0.064 AT to differentiate between non-mineralized $(<0.064 \mathrm{AT})$ and mineralized $(>0.064 \mathrm{AT})$ collagen for subcutaneous injections, which quantified mineralization and allowed for the three-dimensional reconstruction and visualization of the different phases (CTVol software, SkyScan). For each of the datasets, 
the volume percent of mineralized region and fractal dimension were calculated using CTAn software. Three-dimensional images were also reconstructed for all samples.

\section{Histomorphometry}

Subcutaneously injected IDC and IDC-BG gel scaffolds were fixed in $10 \%$ buffered formaldehyde for $24 \mathrm{~h}$, followed by three washes with distilled water before transferring into 70\% ethyl alcohol. Each specimen was processed following routine plastic embedding techniques (McGill Bone Centre). The process included chorological submersion of each specimen in 70, 80, 95 and 100\% ethanol solutions for 12 h periods, followed by Xylene (Fisher) submersion for $3 \mathrm{~h}$, and then embedding in methyl methacrylate, butyl methacrylate (Fisher Scientific), and methyl benzoate (Sigma Aldrich) for $12 \mathrm{~h}$ periods, respectively. The plastic blocks were serially sectioned with a microtome (Leica RM2165, Nussloch, Germany) into $6 \mu \mathrm{m}$ slices along the transverse interface. The presence of mineralized regions was demonstrated by Toluidine blue-von Kossa staining. Adjacent slices were stained with Goldner's Trichrome, to show the collagen fibrils and osteoid presence, and haematoxylin and eosin (H\&E) to provide baseline comparisons. Further sections of those samples which, upon analysis, contained cells with osteoblast and osteoclast-like morphology were stained for alkaline phosphatase (ALP kit, 86c, Sigma Aldrich) and tartrate resistant acid phosphatase (TRAP, 387A, Sigma Aldrich) to further characterise these cells. Sections were briefly deplasticised in acetone for 5 mins then rehydrated through decreasing alcohols to distilled water. They were incubated in staining solution of naphthol AS-BI Alkaline (ALP) or napthol AS-BI phosphoric acid (TRAP) according to the manufacturer's instructions, rinsed with $\mathrm{dH}_{2} \mathrm{O}$ for 3 min and mounted in an aqueous mountant for viewing. 
Images were acquired using a fluorescence microscope (BX51; Olympus Optical Co., Germany) in the bright-field mode and a colour video camera (Soft Imaging System, Germany). Images were taken at $4 \times$ magnification using ImagePro software (Media Cybernetics, USA) and merged to give a composite of the whole slice.

Images were analyzed using the open-source software ImageJ (NIH, USA). The entire scaffold section was reconstructed using a plug-in mosaic, which is consistent with colour images. The amount of mineralization was calculated in the Toluidine blue-von Kossa slices as the area of black stained tissue within the confines of the implant, i.e., a percentage of the region of interest. The images were converted to 8-bit format, and a threshold was adjusted to measure only the black-stained areas of the composite image in terms of percentage. Thresholding was performed by maximum entropy algorithm, and then subjected to the black-white window. The region of interest around the IDC scaffold was selected manually, and the area fraction was calculated in the binary image mode.

\section{Statistical analysis}

Statistical analysis was performed in EXCEL 2010 (Microsoft, USA) using a two-way ANOVA where the main factors are the scaffold type, time point, and gauge number. The level for significance was set at $p=0.05$.

\section{Results and Discussion}

\section{Structural characterization of as-made IDC-BG hybrid gels}

The GAE system (Fig. 1A-C) enabled (in approximately $50 \mathrm{~min}$ ) the scalable fabrication of rod-shaped IDC-BG hybrid gels with defined CFDs, which ranged from approximately 6 to 14 wt\% (Table 1). Along with an increase in CFD with higher gauge numbers, there was a reduction in BG microparticle loading, e.g., from 24 to $20 \mathrm{wt} \%$ in 8G through 14G (for an initial $25 \mathrm{wt} \%$ 
loading). In terms of microstructural properties, schematic Gaussian distribution functions generated from SHG images represented the extent of the overall collagen fibrillar alignment along the longitudinal axis of IDC and IDC-BG scaffolds, which is indicated by the horizontal axis in the left columns of Fig. 2 A-B. SHG images, corroborated by representative SEM micrographs (insets in middle column of Fig. 2), confirmed a decrease in the dispersion of fibril orientation (i.e., the standard deviation of the corresponding Gaussian function) with decreasing needle diameter from 8G (3.4 mm) to 14G (1.6 mm), which represented the opposite ends of the current GAE processing spectrum, and chosen in this study for analysis. Dodt images also confirmed the uniform spatial distribution of BG microparticles deep within IDC-BG scaffolds (Fig. 2C; right column).

The GAE system applies a number forces onto the prefabricated HHC gels during compaction. This is initiated by a steep pressure differential that is applied via the pressure device (e.g., angioplasty inflation device in Fig. 1B) to generate negative pressure, which in turn uses atmospheric pressure as the driving force for compaction. Therefore, the fibrils in HHC gels, which interact through physical entanglements and secondary bonds (i.e., weak, nonchemically cross-linked interactions) experience a two-dimensional compaction process along the circumference of the needle as the gel is aspirated into the orifice of a densification needle. This is thought to provide the driving force behind fibrillar rearrangement and the alignment is induced since the shearing forces exceed the frictional forces established between the randomly arranged fibrils, which are then repositioned along the direction of shear flow.

\section{Mineralization in SBF}

The progress of in vitro mineralization within IDC and IDC-BG gel scaffolds was investigated for up to day 14 in SBF. Fig. 3A illustrates representative SEM micrographs of 
IDC-BG gel scaffolds as-made, and at days 3 and 14 in SBF (Fig. S1 provides more extensive time points) at two length scales. Scaffold immersion in SBF led to the development of apatite crystal-like structure formation on the surface of collagen fibrils, with typical cauliflower morphologies that increased in size and became appeared more granular in shape at longer immersion times [24].

ATR-FTIR spectroscopy and XRD analyses were used to characterize the formed mineral. ATR-FTIR spectra of as-made IDC scaffolds displayed the typical amide absorption bands of collagen fibrils at 1643, 1550 and $1243 \mathrm{~cm}^{-1}$ for Amide I, II and III, respectively (Fig. 3B; left column) [9]. The spectrum of BG exhibited the presence of Si-O-Si vibration mode within the $1000-1100 \mathrm{~cm}^{-1}$ region, and the non-bridging oxygen bond (Si-O-NBO) at around $900 \mathrm{~cm}^{-1}$ (Fig. S2A) [25]. The presence of network modifiers provokes disruption of the glassy network through breaking of the Si-O-Si bonds, thus leading to formation of Si-O-NBO groups. The addition of BG microparticles to IDC yielded a dominant effect of silicate and phosphate vibrations, which masked the side chain vibrations within a wavelength range of $1100-800 \mathrm{~cm}^{-1}$ (Fig. 3B). Since the absorbance of Si-O-Si and $\mathrm{PO}_{4}{ }^{3-}$ groups overlap, the peak at approximately $1000 \mathrm{~cm}^{-1}$ can be attributed to both silicate and phosphate bonds [16]. The peak at $900 \mathrm{~cm}^{-1}$ also shifted to $920 \mathrm{~cm}^{-}$ ${ }^{1}$ in the IDC-BG system, which can be due to a lower amount of Si-O-NBO as well as the formation of Si-OH bonds from BG/water interactions. The weak peak at around $870 \mathrm{~cm}^{-1}$ is also attributed to a double absorbance of $\mathrm{CO}_{3}{ }^{2-}$ and $\mathrm{HPO}_{4}{ }^{2-}$ groups [9].

Post immersion in SBF, the FTIR spectra of IDC-BG scaffolds showed a slight shift in the wavenumber of amide I absorption along with an increase in the absorption bands of $\mathrm{PO}_{4}{ }^{3-}$ and $\mathrm{CO}_{3}{ }^{2-}$ at 1022 and $874 \mathrm{~cm}^{-1}$, respectively (Fig. 3B); attributable to the formation of crystalline CHA within the scaffolds [9]. In addition, there was a progressive increase in absorbance of the 
Si-O-Si peaks, at 1100 and $803 \mathrm{~cm}^{-1}$ [26]. The XRD diffractographs of IDC-BG scaffolds indicated the progressive intensification of the characteristic peaks of hydroxyapatite with time in SBF, and by day 14, the broad diffraction peak at $2 \theta \sim 20^{\circ}$ in the as-made gels (corresponding to collagen structural characteristics, along with the amorphous nature of BG; Fig S2) was replaced by peaks such as $2 \theta \sim 32^{\circ}$, indicative of formation of low crystalline apatite (Fig. 3C; right column). XRD analysis (in line with ATR-FTIR), indicated similar trends for CHA formation in IDC-BG gel scaffolds produced through 8G and 14G needles (Fig. S2). EDAX analysis confirmed the co-presence of $\mathrm{Si}$, Ca and $\mathrm{P}$ in the mineralized IDC-BG scaffolds (Fig. S2). IDC scaffolds, on the other hand, only showed indications of CHA formation at day 7 in SBF, followed by the presence of detectable CHA at day 14 (Fig. 3B-C, left columns) [15].

Typical tensile stress-strain curves generated for IDC and IDC-BG gel scaffolds (produced through $14 \mathrm{G}$ ), as-made, and as a function of time in SBF, provided an indication of the effect of mineralization on gel scaffold mechanical properties (Fig. S3). As-made IDC and IDC-BG gels demonstrated typical toe, linear and failure regions associated with dense collagen gels [9, 16, 17, 27]. The progressive increase in mineralization in IDC-BG gels triggered a transition from soft-to-hard tissue-like response to tensile force, as indicated by the stress-strain curve tending towards the y-axis (Fig. S3B); indicating an increase in the stiffness of the scaffolds. This was in contrast to minor changes in stress-strain curves of the IDC gel scaffolds over time in SBF (Fig. S3A) [9].

\section{In vivo mineralization}

To extend upon the above findings and evaluate the feasibility of GAE in a surgical context, acellular gel scaffolds were subcutaneously injected into the flanks of adult rats. At days 7 and 21 following subcutaneous injection, rats were euthanized and the scaffolds were 
extracted. In general, post-implanted IDC and IDC-BG scaffolds exhibited a planar morphology most likely due to material compression under the weight of the animal during normal waking activity. Scaffolds were well retained within the subcutaneous injection site and did not appear to shift laterally or along the rostro-caudal axis. IDC-BG scaffolds were noticeably more rigid than IDC scaffolds and at day 21 appeared qualitatively more resistant to deformation during sample isolation and handling than those collected at day 7. In addition, at day 21, the IDC and IDC-BG scaffolds were often bonded to subcutaneous connective tissues with no gross signs of inflammation. In some cases, tissue attachment was present along the dorsal surface of the scaffold, where the attachment could not be manually dissociated during sample collection.

Compared to as-made gels, and subcutaneously implanted IDC gels, three-dimensional reconstruction of micro-CT scans of IDC-BG gel scaffolds at days 7 and 21 post implantation illustrated an increase in the attenuation of x-ray; confirming the presence of an increasingly dense material, i.e., mineralization (Fig. 4A). Analysis of micro-CT data confirmed the significant ( $p<0.05$; where main factors are the implant type and dissection time) rapid increase in mineralized volume percent of collagen in IDC-BG scaffolds (Table 3). At day 21, IDC gel scaffolds exhibited a mineralized phase of 5-15\%, indicating spontaneous deposition of calcium ions on the surface of collagen fibrils. In contrast, the mineralized phase in IDC-BG gel scaffolds was significantly higher, reaching $40-50 \%$. There were no significant $(p>0.05)$ differences in the extent of mineralization between similar groups generated from the two different gauge numbers. Furthermore, there was a significant $(p<0.05$; where main factors are the implant type and dissection time) decrease in fractal dimension in IDC-BG at day 21, suggesting an increase in the stiffness of the scaffolds [28]. 
ATR-FTIR and XRD analyses characterized the mineralization of subcutaneously injected IDC and IDC-BG gels (Fig. 4 B\&C, respectively). ATR-FTIR spectra indicated the progressive mineralization of IDC-BG gels, as the absorbance peaks associated with phosphate and carbonate bonds emerged at days 7 and 21, comparable to those detected in vitro (Fig. S2). XRD diffractographs of explanted IDC-BG scaffolds showed the main hydroxyapatite peak around $2 \theta$ $\sim 32^{\circ}$ at day 7 , in addition to a broad hump of the collagen matrix. At day 21 post injection, additional peaks were exhibited that corresponded with hydroxyapatite, similar to those demonstrated in vitro. The evolution of XRD patterns confirmed the rapid rate of mineralization in IDC-BG scaffolds and was in contrast to those of IDC scaffolds, which lacked the diffraction peaks associated with hydroxyapatite.

Standard Toluidine blue-von Kossa staining showed that both IDC and IDC-BG scaffolds contained mineralized regions (black staining; Fig. 5). However, the pattern of mineralization was different in each group, where it generally occurred in the centre of IDC scaffolds, not reaching the surrounding capsule, and in contrast to that in IDC-BG, where mineralisation was observed around the edges and up to the sub-capsular space (Fig. 6). Quantification of von Kossa staining indicated that the mineralized area percent in explanted IDC-BG was significantly greater ( $p<0.05$; where main factors were the implant type and dissection time) than in IDC scaffolds (Table 4), as indicated by micro-CT analysis (Table 3). On the other hand, the mineralized area percent was not sensitive to the gauge number $(p=0.13$; where main factors were the gauge number and dissection time), which also correlated with the micro-CT analysis. Goldner's Trichrome stained histological sections confirmed the presence of collagen-rich regions (green fibril staining in Fig. 5) in both IDC and IDC-BG scaffolds, along with directional orientation of collagen fibrils within the gel scaffolds. 
Histological analysis of the IDC and IDC-BG gel scaffolds showed that all implants became encapsulated in the subcutaneous tissue. The capsule thickness varied both between and within samples although in general it was approximately 1-2 cells thick (Fig. 6). Cell infiltration was present in both groups, beginning at the edges of the injected scaffolds and penetrating between collagen fibrils and along any channels or folds in the scaffolds. Cell infiltration was more evident in the IDC-BG group and increased with time. At day 21, cell infiltration into IDC was similar to that seen in IDC-BG scaffolds at day 7 and was characterised by individual cells penetrating into the dense collagen network, with sections remaining un-colonized (Fig. 6). By contrast, at day 21, cell infiltration in IDC-BG had progressed to islands of disorganized granulation tissue between denser areas of un-colonized scaffold that did not seem to be related to needle gauge size. Furthermore, in the majority of samples in this group, this tissue showed significant signs of remodelling with cells resembling osteoblasts, osteoclast-like cells lining the scaffold/tissue interface, the presence of osteoid and areas of tissue which had the appearance of woven bone (Figs. 6 and S4). Upon further analysis, it was confirmed that cells in these areas expressed appropriate markers for osteoblasts (ALP) and osteoclasts (TRAP) (Fig. S4).

This organization of tissue was accompanied by significant neovascularization, the early signs of which were visible at day 7 in IDC-BG (Figs. 6 and S4). There was no evidence of vascularization in the IDC group at day 7 although vessels were seen in this group at day 21 . Furthermore, in some samples, the BG particles formed local agglomerates that were visible both at days 7 and 21. At the earlier time point, this invoked a foreign body giant cell response, but by day 21 the agglomerates seemed to be the site of osteoid formation, as confirmed by H\&E and Goldner's Trichrome staining (Fig. 6). No foreign body reaction was seen in the IDC groups. 
Much of the histological findings in this study are supported by previous reports, where others have demonstrated similar capsule formation and/or cell infiltration into poly(3hydroxybutyrate)/BG composite foams [29], poly(e-caprolactone/D,L-lactide)/BG scaffolds [30], nanoporous bioactive glass scaffolds [31] and bioactive glass particles [32] when implanted subcutaneously either in rats [29, 30,32] or New Zealand white rabbits [31]. Similarly, the proangiogenic properties of bioactive glasses have been widely reported, where they have been shown to induce increased vascular endothelial growth factor (VEGF) and basic fibroblast growth factor expression by endothelial cells or fibroblasts [14, 33, 34] and enhanced tubule formation and branching of endothelial cell cultures [14, 33], in vitro. In vivo, the presence of BG in composites of poly(D,L-lactide-co-glycolide) [35] and polyglycolic acid [34] and glasscoated collagen [8] was associated with increased vascularization as measured by blood vessel count or haemoglobin content. Even the observation of BG agglomerations and giant cell formation has been reported elsewhere [29, 30]. There is evidence that BG has direct osteogenic and mineralogenic effects in vitro through the promotion of ALP activity and the enhanced expression of osteogenic genes such as osteocalcin, RUNX2, BMP2, BMP6, osterix, bone sialoprotein, osteonectin and collagen I [36-39], yet, while bone induction in an ectopic intramuscular site has been previously reported by BG generated glass-ceramic scaffolds [40], no previous study has reported BG-induced bone formation in a subcutaneous site without the addition of osteogenic cells (despite implantation times ranging from 7 to 60 days).

In discussing osteoinductive calcium phosphates, Habibovic and de Groot [41] described the conditions required for osteoinduction to occur in the absence of strong evidence of sequestered bone morphogenetic protein involvement (the classical mechanism of osteoinduction). They suggest that the required material properties are the presence of macro- 
and micro-pores post-implantation and the formation of a CHA layer which could act either as a trigger for osteogenic differentiation of local stem cells or could include growth factors that drive the process. Certainly, IDC-BG gels satisfy both of these criteria with folds, channels and pores allowing cell infiltration and von Kossa staining demonstrating early mineralization of the scaffold. Furthermore, given the proangiogenic and osteogenic properties of BG [36, 39, 42], it is probable that both material and biological characteristics of the IDC-BG scaffold are contributing to the phenomenon of osteoinduction: in addition to the direct stimulation of osteogenesis and mineralisation that may be stimulated by BG, angiogenesis and osteogenesis are closely related and the presence of VEGF has been shown to enhance bone healing in orthotopic sites [43]. Indeed, the sites of tissue remodelling and woven bone-like tissue reported here were always co-localised with multiple blood vessels. Habibovic and de Groot [41] also suggested that osteoinduction in rodent subcutaneous models is rare as this is a less inductive site than the intramuscular sites usually chosen in larger animals such as dog and goat. Therefore, the results of the in vivo models described above, where good cell infiltration and vascularization of BG/composite scaffolds was reported, but no bone formation was seen, would support this suggestion and makes the results reported here more promising, particularly as evidence would suggest that increased osteoinductive properties of a scaffold will translate into increased bone formation in an orthotopic site [41].

SHG imaging of explanted scaffolds indicated remodelling within the mineralized regions (Fig. 7; Fig. S5 provides larger views), where small mineral nodules were percolated into the IDC-BG scaffolds over time. Collagen fibrils in the localized mineralization regions were observed to be highly aligned and bundle shaped, while gradually transitioning to more isotropic fibrils in non-mineralized regions with visible disruption in the orientation of collagen fibrils 
across the transition regions. It has been previously shown that the culturing of pre-osteoblasts, three-dimensionally seeded in BG incorporated dense collagen gels of random fibrillar orientation, resulted in their preferential orientation and adjacent to highly aligned, mineralized collagen fibrils [9]. More recently, it was demonstrated that the osteoblastic differentiation of mesenchymal stem cells and matrix mineralization was accelerated when seeded in aligned IDC gels compared to similar gels of random fibrillar orientation [18]. The sparse distributions of the SHG signal intensity (i.e., a mixture of very bright and dark regions in Fig. 7C) also confirmed their planar shape as the incident laser beams were linearly polarized. This was in contrast to the more coherent intensity distribution in the non-mineralized regions, or in as-made gels (Fig. 2). The Dodt images provide further evidence for cellular infiltration and mineralization within the IDC-BG scaffolds (Figs. 7 and S5), suggesting that this deep tissue imaging technique may provide a quantitative tool to assess the progression of mineralization [23].

In contrast to the as-made scaffolds, where collagen fibrils aligned along the longitudinal direction (Fig. 2), collagen fibrils of explanted scaffolds showed less directionality (Fig. 7), demonstrating parallel alignment in the edges and more isotropically distributed in central regions (Fig. S5). SHG imaging also indicated that the distribution in collagen orientation of explanted 8G and 14G IDC scaffolds were similar. Attributable to the symmetry in physiological conditions between the two flank sites, the collagen microstructure remodelled towards a similar configuration in both scaffolds. This would justify the low statistical difference in histomorphometry and micro-CT measurements between the two gauge numbers and may be due to the non-confined positioning of the scaffold when subcutaneously implanted, evolving toward a flattened disk shape and altering the initial CFD value. It is anticipated that the physiological conditions will be different in an orthotropic site. 


\section{Potential outlook for in situ bone defect injection}

Since a substantial fraction of bone defects do not heal properly using bone autografts, the gold standard treatment, alternative strategies have relied on the osteoconductive or osteoinductive capabilities of an implanted tissue such as an allograft or synthetic materials such as bioceramics [44]. On the other hand, bone tissue engineering is diversifying toward biologically derived biodegradable materials and their integration with therapeutic cells and bioactive factors. With the current limitations of orthobiologic products [7], there is a need for the development of new biomaterial scaffolds. The utilization of a GAE system in this study, rapidly and simply generated cylindrical-shaped IDC-BG hybrid gels with controllable CFD values and microstructural properties, thus potentially enabling the minimally invasive delivery of more stable dense collagen gel scaffolds into a surgical site, something which has previously been challenging [45]. To reduce large tissue voids and gaps for bone repair, IDC gel fabrication can be readily scaled, with little increase in processing time, for example, by using larger cylindrical moulds to accommodate larger volumes of precursor HHC gels. As such, the GAE injectable hydrogel system could potentially address a number of current issues including cost and risks associated with multiple treatments. Moreover, the addition of BG to the gel seemed to infer osteoinductive properties that have not previously been reported. Most synthetic bone substitutes are osteoconductive only and the addition of an osteoinductive capability would suggest the possibility of enhanced clinical performance with this scaffold. Therefore, future studies will be required to investigate the injection of IDC-BG scaffolds into bone defects.

\section{Conclusions}

A GAE system was used to rapidly fabricate IDC-BG gel scaffold that demonstrated bioactive and osteoinductive properties, as indicated by the formation of CHA in SBF and bone- 
like material in an ectopic environment, in vivo. SHG imaging demonstrated collagen remodelling associated with mineralization in subcutaneously injected IDC-BG scaffolds. The proposed IDC-BG hybrid gel potentially offers an efficient approach for therapeutics requiring an injectable delivery of a bone graft and may provide enhanced clinical performance over currently available alternatives.

\section{Acknowledgements}

Funding of CIHR, NSERC, CFI, Quebec MEIE, FQRNT, McGill University Faculty of Engineering Gerald Hatch Faculty Fellowship and MEDA are gratefully acknowledged. The authors would like to acknowledge the Comparative Medicine and Animal Resources Centre (McGill Life Sciences Complex, McGill University) as well as Imaging and Molecular Biology Platform center (Pharmacology and Therapeutics Department, McGill University) for their technical supports. 


\section{References:}

[1] Giannoudis PV, Dinopoulos H, Tsiridis E. Bone substitutes: an update. Injury. 2005;36:S20S7.

[2] Bohner M. Resorbable biomaterials as bone graft substitutes. Materials Today. 2010;13:2430.

[3] Parikh S. Bone graft substitutes: past, present, future. Journal of Postgraduate Medicine. 2002;48:142.

[4] Hollinger JO, Einhorn TA, Doll B, Sfeir C. Bone tissue engineering: CRC Press; 2004.

[5] Heinemann S, Heinemann C, Ehrlich H, Meyer M, Baltzer H, Worch H, et al. A novel biomimetic hybrid material made of silicified collagen: perspectives for bone replacement. Advanced Engineering Materials. 2007;9:1061-8.

[6] Habibovic P, Bassett DC, Doillon CJ, Gerard C, McKee MD, Barralet JE. Collagen biomineralization in vivo by sustained release of inorganic phosphate ions. Advanced Materials. 2010;22:1858-62.

[7] Shields LB, Raque GH, Glassman SD, Campbell M, Vitaz T, Harpring J, et al. Adverse effects associated with high-dose recombinant human bone morphogenetic protein-2 use in anterior cervical spine fusion. Spine. 2006;31:542-7.

[8] Andrade ÂL, Andrade SP, Domingues RZ. In vivo performance of a sol-gel glass-coated collagen. Journal of Biomedical Materials Research Part B: Applied Biomaterials.

2006;79B:122-8.

[9] Marelli B, Ghezzi CE, Mohn D, Stark WJ, Barralet JE, Boccaccini AR, et al. Accelerated mineralization of dense collagen-nano bioactive glass hybrid gels increases scaffold stiffness and regulates osteoblastic function. Biomaterials. 2011;32:8915-26.

[10] Hench LL, Greenspan D. Interactions between bioactive glass and collagen: A review and new perspectives. Journal of the Australian Ceramics Society. 2013;49:1-40.

[11] Sarker B, Hum J, Nazhat SN, Boccaccini AR. Combining collagen and bioactive glasses for bone tissue engineering: a review. Advanced Healthcare Materials. 2015;4:176-194.

[12] Xu C, Su P, Chen X, Meng Y, Yu W, Xiang AP, et al. Biocompatibility and osteogenesis of biomimetic Bioglass-Collagen-Phosphatidylserine composite scaffolds for bone tissue engineering. Biomaterials. 2011;32:1051-8.

[13] Chen QZ, Ahmed I, Knowles JC, Nazhat SN, Boccaccini AR, Rezwan K. Collagen release kinetics of surface functionalized 45S5 Bioglass ${ }^{\circledR}$-based porous scaffolds. Journal of Biomedical Materials Research Part A. 2008;86A:987-95.

[14] Leu A, Leach JK. Proangiogenic potential of a collagen/bioactive glass substrate. Pharmaceutical Research. 2008;25:1222-9.

[15] Marelli B, Ghezzi CE, Barralet JE, Nazhat SN. Collagen gel fibrillar density dictates the extent of mineralizationin vitro. Soft Matter. 2011;7:9898-907.

[16] Marelli B, Ghezzi CE, Barralet JE, Boccaccini AR, Nazhat SN. Three-dimensional mineralization of dense nanofibrillar collagen-Bioglass hybrid scaffolds. Biomacromolecules. 2010;11:1470-9.

[17] Brown RA, Wiseman M, Chuo CB, Cheema U, Nazhat SN. Ultrarapid engineering of biomimetic materials and tissues: fabrication of nano- and microstructures by plastic compression. Advanced Functional Materials. 2005;15:1762-70. 
[18] Marelli B, Ghezzi CE, James-Bhasin M, Nazhat SN. Fabrication of injectable, cellular, anisotropic collagen tissue equivalents with modular fibrillar densities. Biomaterials.

2015;37:183-93.

[19] Haruhiko N. Partial specific volume of collagen. Journal of Biochemistry. 1972;71:699-703.

[20] Chen Q, Boccaccini A. Poly (D, L-lactic acid) coated 45S5 Bioglass ${ }^{\circledR}$-based scaffolds:

Processing and characterization. Journal of Biomedical Materials Research Part A. 2006;77:445-

57.

[21] Kokubo T. Protocol for preparing simulated body fluid SBF. Department of Material

Chemistry, Graduate School of Engineering, Kyoto University, Japan; 1990.

[22] Miri AK, Tripathy U, Mongeau L, Wiseman PW. Nonlinear laser scanning microscopy of human vocal folds. The Laryngoscope. 2012;122:356-63.

[23] Dodt H-U, Eder M, Frick A, Zieglgänsberger W. Precisely localized LTD in the neocortex revealed by infrared-guided laser stimulation. Science. 1999;286:110-3.

[24] Rezwan K, Chen QZ, Blaker JJ, Boccaccini AR. Biodegradable and bioactive porous polymer/inorganic composite scaffolds for bone tissue engineering. Biomaterials. 2006;27:341331.

[25] Serra J, González P, Liste S, Serra C, Chiussi S, León B, et al. FTIR and XPS studies of bioactive silica based glasses. Journal of Non-Crystalline Solids. 2003;332:20-7.

[26] Cerruti M, Greenspan D, Powers K. Effect of $\mathrm{pH}$ and ionic strength on the reactivity of Bioglass ${ }^{\circledR}$ 45S5. Biomaterials. 2005;26:1665-74.

[27] Abou Neel EA, Cheema U, Knowles JC, Brown RA, Nazhat SN. Use of multiple unconfined compression for control of collagen gel scaffold density and mechanical properties.

Soft Matter. 2006;2:986-92.

[28] Millard J, Augat P, Link TM, Kothari M, Newitt DC, Genant HK, et al. Power spectral analysis of vertebral trabecular bone structure from radiographs: orientation dependence and correlation with bone mineral density and mechanical properties. Calcified Tissue International. 1998;63:482-9.

[29] Misra SK, Ansari TI, Valappil SP, Mohn D, Philip SE, Stark WJ, et al. Poly (3hydroxybutyrate) multifunctional composite scaffolds for tissue engineering applications. Biomaterials. 2010;31:2806-15.

[30] Meretoja VV, Tirri T, Malin M, Seppälä JV, Närhi TO. Ectopic bone formation in and soft-tissue response to P (CL/DLLA)/bioactive glass composite scaffolds. Clinical Oral Implants Research. 2014;25:159-64.

[31] Wang S, Kowal TJ, Marei MK, Falk MM, Jain H. Nanoporosity significantly enhances the biological performance of engineered glass tissue scaffolds. Tissue Engineering Part A. 2013;19:1632-40.

[32] da Cruz ACC, Pochapski MT, Tramonti R, da Silva JCZ, Antunes AC, Pilatti GL, et al. Evaluation of physical-chemical properties and biocompatibility of a microrough and smooth bioactive glass particles. Journal of Materials Science: Materials in Medicine. 2008;19:2809-17. [33] Day RM. Bioactive glass stimulates the secretion of angiogenic growth factors and angiogenesis in vitro. Tissue engineering. 2005;11:768-77.

[34] Day RM, Boccaccini AR, Shurey S, Roether JA, Forbes A, Hench LL, et al. Assessment of polyglycolic acid mesh and bioactive glass for soft-tissue engineering scaffolds. Biomaterials. 2004;25:5857-66. 
[35] Day RM, Maquet V, Boccaccini AR, Jérôme R, Forbes A. In vitro and in vivo analysis of macroporous biodegradable poly ( $\mathrm{D}$, L-lactide-co-glycolide) scaffolds containing bioactive glass. Journal of Biomedical Materials Research Part A. 2005;75:778-87.

[36] Hoppe A, Güldal NS, Boccaccini AR. A review of the biological response to ionic

dissolution products from bioactive glasses and glass-ceramics. Biomaterials. 2011;32:2757-74.

[37] Tousi NS, Velten MF, Bishop TJ, Leong KK, Barkhordar NS, Marshall GW, et al.

Combinatorial effect of $\mathrm{Si}^{4+}, \mathrm{Ca}^{2+}$, and $\mathrm{Mg}^{2+}$ released from bioactive glasses on osteoblast osteocalcin expression and biomineralization. Materials Science and Engineering: C. 2013;33:2757-65.

[38] Alves EG, Serakides R, Rosado IR, Pereira MM, Ocarino NM, Oliveira HP, et al. Effect of the ionic product of bioglass 60s on osteoblastic activity in canines. BMC Veterinary Research. 2015;11:1.

[39] El-Gendy R, Yang XB, Newby PJ, Boccaccini AR, Kirkham J. Osteogenic differentiation of human dental pulp stromal cells on 45S5 Bioglass ${ }^{\circledR}$ based scaffolds in vitro and in vivo. Tissue Engineering Part A. 2012;19:707-15.

[40] Yuan H, de Bruijn JD, Zhang X, van Blitterswijk CA, de Groot K. Bone induction by porous glass ceramic made from Bioglass ${ }^{\circledR}(45 S 5)$. Journal of Biomedical Materials Research. 2001;58:270-6.

[41] Habibovic P, de Groot K. Osteoinductive biomaterials—properties and relevance in bone repair. Journal of Tissue Engineering and Regenerative Medicine. 2007;1:25-32.

[42] Gorustovich AA, Roether JA, Boccaccini AR. Effect of bioactive glasses on angiogenesis: a review of in vitro and in vivo evidences. Tissue Engineering Part B: Reviews. 2009;16:199-207. [43] Clarke S, Hoskins N, Jordan G, Marsh D. Healing of an ulnar defect using a proprietary TCP bone graft substitute, JAX ${ }^{\mathrm{TM}}$, in association with autologous osteogenic cells and growth factors. Bone. 2007;40:939-47.

[44] Poinern GEJ, Brundavanam RK, Thi Le X, Nicholls PK, Cake MA, Fawcett D. The synthesis, characterisation and in vivo study of a bioceramic for potential tissue regeneration applications. Scientific Reports. 2014;4:1-9.

[45] Sadiasa A, Sarkar SK, Franco RA, Min YK, Lee BT. Bioactive glass incorporation in calcium phosphate cement-based injectable bone substitute for improved in vitro biocompatibility and in vivo bone regeneration. Journal of Biomaterials Applications. 2014;28:739-56. 


\section{Figure Captions:}

\section{Figure 1. IDC and IDC-BG gel scaffold production and subcutaneous injection through}

GAE. (A) Schematic representation of the IDC-BG gel scaffold fabrication. Time marks illustrate rapidity of the process (total time is less than $50 \mathrm{~min}$ ). (B) Schematic of GAE system showing its precursor highly-hydrated gel in a 48 well plate before and during processing. (C) In vivo study showing fabrication of the precursor and aspiration process in a 14G needle in the procedure room; and (D) placement of one hybrid gel implant in the left flank of the rat.

Figure 2. Collagen fibrillar orientation. (A) SHG images (left and middle columns) and Dodt image (right column) of an IDC gel scaffold fabricated by 8G needle, (B) an IDC gel scaffold fabricated by $14 \mathrm{G}$ needle, and (C) an IDC-BG gel scaffold fabricated by $14 \mathrm{G}$ needle. SHG and Dodt images were generated for the same chosen region. The Gaussian distributions in (B) \& (C) indicated the of overall fibrillar orientation in the IDC-based gels produced through the two gauge numbers. Insets in middle column showing representative SEM micrographs. White arrows in (C) indicate BG microparticles within the IDC-BG gel scaffold.

Figure 3. Mineralization in SBF. (A) SEM micrographs of IDC-BG gel scaffolds fabricated by 14G needle as-made, and at days 3 and 14 in SBF. (B) ATR-FTIR spectra of IDC (left column) and IDC-BG (right column) gel scaffolds fabricated by 14G needle as a function of time in SBF. (C) XRD diffractographs of IDC (left column) and IDC-BG (right column) gel scaffolds fabricated by $14 \mathrm{G}$ needle as a function of time in SBF. The standard peaks for hydroxyapatite, based on the International Centre for Diffraction Data, are shown in the right column.

Figure 4. In vivo mineralization. (A) Micro-CT (based on the attenuation values) images of representative IDC and IDC-BG gel scaffolds as-made and at days 7 and 21 post subcutaneous injections. (B) ATR-FTIR spectra of explanted gel scaffolds in right flank and (C) both flanks at 
day 21. (D) XRD patterns of explanted gel scaffolds in right flank with standard hydroxyapatite, and (E) both flanks at day 21.

Figure 5. Gross histological analysis of subcutaneously injected scaffolds. Histology images of representative IDC and IDC-BG gel scaffolds as-made and at days 7 and 21 post subcutaneous injection. High-power insets in as-made IDC and IDC-BG gels indicate the distribution of collagen fibrils (Goldner’s Trichrome) and BG microparticles (von Kossa-Toluidine blue: yellow arrows).

Figure 6. Histological analysis of implants. Top row: Capsule formation was evident around the scaffolds at day 7. In most instances the capsule was 1-2 cells thick (arrows). Mineralized tissue (black stained) did not quite reach the capsular membrane in the IDC group, but reached the sub-capsular layers in the IDC-BG group. Second row: Cell infiltration into the scaffolds was indicated (arrows), which was less pronounced in the IDC groups at both days 7 and 21. Cells had penetrated into the centre of IDC-BG at day 7 and by day 21 islands of cells had developed within the scaffold (white arrow) and there was separation of the collagen fibrils (red arrow). Third row: Neovascularization was evident within the confines of the scaffold. No blood vessels were seen in the IDC group at day 7, but some small vessels were visible at the edges of the IDC-BG scaffolds (arrows). By day 21, there was significant neovascularization in both IDC and IDC-BG scaffolds in the areas of tissue/cell penetration. Fourth row: The BG particles formed local agglomerations (black arrows) in some instances, which stimulated a foreign body giant cell response (white arrows) at day 7 but at day 21 seemed to be the site of osteoid formation (red arrows). Fifth row: Further evidence of scaffold remodelling and osteoinduction in the IDCBG group at day 21 was observed with the presence of cells phenotypically similar to both osteoblasts (column 1, confirmed by ALP staining shown in Fig. S4) and osteoclasts (confirmed 
by TRAP staining shown in Fig. S4), at the scaffold/tissue interface (column 2), and remodelling of the collagenous material (column 3) into tissue with the appearance of woven bone (column 4). Key: $\mathrm{S}=$ scaffold, $\mathrm{H} \& \mathrm{E}=$ haematoxylin and eosin, $\mathrm{TB} \& \mathrm{vK}=$ Toluidine blue and von Kossa, G Tri = Goldner's Trichrome.

Figure 7. Imaging of collagen fibrils in explants. (A) SHG and Dodt images of subcutaneously injected IDC gel at day 7, (B) IDC-BG at day 7, and (C) IDC-BG at day 21. White arrows in (A) and (B) indicate the infiltration of particles and cells, respectively. 
Table 1. The equivalent collagen fibrillar density (CFD; obtained from IDC scaffolds and assuming that no collagen was lost during gelation and the GAE fabrication process) and BG mass and volume percent in IDC-BG gel scaffolds for two distinct collagen-BG mass ratios $(N=$ 3).

\begin{tabular}{|c|c|c|c|c|c|}
\hline \multirow{2}{*}{$\begin{array}{l}\text { Initial collagen-BG } \\
\text { mass ratio (dry wt \%) }\end{array}$} & Gauge number & 8G & 10G & $12 G$ & $14 G$ \\
\hline & Diameter (mm) & 3.43 & 2.69 & 2.16 & 1.60 \\
\hline $100-0$ & CFD (wt \%) & $5.74 \pm 0.14$ & $8.30 \pm 0.75$ & $11.04 \pm 0.94$ & $13.82 \pm 2.06$ \\
\hline \multirow[t]{3}{*}{$75-25$} & $\begin{array}{l}\text { Solid mass } \\
\text { content (wt \%) }\end{array}$ & $7.12 \pm 0.17$ & $10.15 \pm 0.92$ & $13.34 \pm 1.14$ & $16.59 \pm 2.47$ \\
\hline & $\begin{array}{l}\text { Practical BG } \\
\text { mass (wt \%) }\end{array}$ & $24.06 \pm 0.77$ & $22.24 \pm 0.47$ & $20.87 \pm 0.83$ & $20.03 \pm 0.85$ \\
\hline & $\begin{array}{l}\text { Practical BG } \\
\text { volume (vol \%) }\end{array}$ & 0.49 & 0.74 & 0.85 & 1.46 \\
\hline \multirow[t]{3}{*}{$50-50$} & $\begin{array}{l}\text { Solid mass } \\
\text { content (wt \%) }\end{array}$ & $8.59 \pm 0.21$ & $12.23 \pm 1.10$ & $16.02 \pm 1.36$ & $19.99 \pm 2.98$ \\
\hline & $\begin{array}{l}\text { Practical BG } \\
\text { mass (wt \%) }\end{array}$ & $49.66 \pm 0.71$ & $47.31 \pm 0.42$ & $45.13 \pm 0.80$ & $44.72 \pm 0.82$ \\
\hline & $\begin{array}{l}\text { Practical BG } \\
\text { volume (vol \%) }\end{array}$ & 1.63 & 2.72 & 3.67 & 4.65 \\
\hline
\end{tabular}


Table 2. The number of animals assigned for each treatment as well as the characterization types.

\begin{tabular}{|c|c|c|c|c|c|}
\hline \multirow{2}{*}{$\begin{array}{l}\text { Animal } \\
\text { number }\end{array}$} & \multirow{2}{*}{$\begin{array}{l}\text { Implant } \\
\text { type }\end{array}$} & \multirow{2}{*}{$\begin{array}{c}\text { Gauge } \\
\text { number }\end{array}$} & \multirow{2}{*}{$\begin{array}{l}\text { Dissection } \\
\text { time (day) }\end{array}$} & \multicolumn{2}{|c|}{ Characterization type } \\
\hline & & & & micro-CT/histology & chemical analysis \\
\hline \multirow[t]{2}{*}{$1-3$} & \multirow[t]{4}{*}{ IDC } & 8G & \multirow[b]{2}{*}{7} & 3 & 3 \\
\hline & & 14G & & 3 & 3 \\
\hline \multirow[t]{2}{*}{ 4-6 } & & 8G & \multirow{2}{*}{21} & 3 & 3 \\
\hline & & 14G & & 3 & 3 \\
\hline \multirow[t]{2}{*}{$7-12$} & \multirow[t]{2}{*}{ IDC-BG } & $8 G$ & \multirow{2}{*}{7} & 6 & 6 \\
\hline & & $14 G$ & & 6 & 6 \\
\hline \multirow[t]{2}{*}{ 13-18 } & & 8G & \multirow{2}{*}{21} & 6 & 6 \\
\hline & & 14G & & 6 & 6 \\
\hline
\end{tabular}


Table 3. Quantification of micro-CT analysis. The presence of BG particles in the subcutaneously injected hybrid gel scaffolds increased both the mineralized volume percent and fractal dimension ( $p<0.05$; by a two-way ANOVA where the factors are implant type and dissection time).

Implant Dissection time (day) Gauge number Mineralized volume percent (\%) Fractal dimension

\begin{tabular}{ccccc}
\hline \hline IDC & as-made & $8 G$ & 0 & $2.96 \pm 0.02$ \\
& & $14 G$ & 0 & $2.95 \pm 0.01$ \\
\hline IDC-BG & as-made & $8 G$ & 0 & $2.96 \pm 0.02$ \\
& & $14 G$ & 0 & $2.94 \pm 0.01$ \\
\hline IDC & 7 & $8 G$ & $1.15 \pm 1.04$ & $2.95 \pm 0.01$ \\
& 21 & $14 G$ & $1.81 \pm 0.81$ & $2.97 \pm 0.02$ \\
& & $8 G$ & $11.49 \pm 7.61$ & $2.92 \pm 0.04$ \\
& 7 & $14 G$ & $10.31 \pm 5.04$ & $2.89 \pm 0.03$ \\
\hline IDC-BG & & $14 G$ & $9.73 \pm 1.91$ & $2.93 \pm 0.03$ \\
& 21 & $8 G$ & $11.30 \pm 3.29$ & $2.92 \pm 0.03$ \\
& & $14 G$ & $40.33 \pm 7.70$ & $2.80 \pm 0.05$ \\
& & & $44.37 \pm 6.03$ & $2.69 \pm 0.06$
\end{tabular}


Table 4. Quantification of von Kossa staining of histological sections. The presence of BG particles in the subcutaneously injected hybrid gels significantly ( $p<0.05$; by a two-way ANOVA where the factors are implant type and dissection time) increased the mineralized area percent.

\begin{tabular}{cccc} 
Implant & Dissection time (day) & Gauge number & Mineralized area percent (\%) \\
\hline \hline IDC & 7 & $8 G$ & $2.92 \pm 2.81$ \\
& 21 & $14 G$ & $2.86 \pm 2.14$ \\
& & $8 G$ & $13.14 \pm 10.90$ \\
& 7 & $14 G$ & $8.58 \pm 7.53$ \\
\hline IDC-BG & 21 & $8 G$ & $15.91 \pm 2.65$ \\
& & $14 G$ & $16.12 \pm 2.32$ \\
& & $8 G$ & $53.53 \pm 9.61$ \\
& & $14 G$ & $47.23 \pm 5.13$
\end{tabular}

